Faculty of Mathematical Sciences

\section{University of Twente}

University for Technical and Social Sciences
P.O. Box 217

7500 AE Enschede

The Netherlands

Phone: +31-53-4893400

Fax: +31-53-4893114

Email: memo@math.utwente.nl

\author{
Memorandum No. 1510 \\ Portcontrolled Hamiltonian representation \\ of distributed parameter systems \\ B.M.J. MASChKE ${ }^{1}$ AND A.J. VAN DER SCHAFT
}

JANUARY 2000

ISSN 0169-2690

\footnotetext{
${ }^{1}$ Laboratoire d'Automatisme Industriel, Conservatoire National des Arts et Métiers, Paris, France
} 


\title{
PORT CONTROLLED HAMILTONIAN REPRESENTATION OF DISTRIBUTED PARAMETER SYSTEMS
}

\author{
B.M.J.Maschke*,** A.J. van der Schaft* \\ * Faculty of Mathematical Sciences, Department of Systems, \\ Signals and Control, University of Twente, P.O.Box 217, \\ 7500 AE Enschede, The Netherlands, e-mail: \\ \{maschke,a.j.vanderschaft\}@math.utwente.nl \\ ** Laboratoire d'Automatisme Industriel, Conservatoire National \\ des Arts et Métiers, Paris, France
}

\begin{abstract}
A port controlled Hamiltonian formulation of the dynamics of distributed parameter systems is presented, which incorporates the energy flow through the boundary of the domain of the system, and which allows to represent the system as a boundary control Hamiltonian system. This port controlled Hamiltonian system is defined with respect to a Dirac structure associated with the exterior derivative and based on Stokes' theorem. The definition is illustrated on the examples of the telegrapher's equations, Maxwell's equations and the vibrating string.
\end{abstract}

Keywords: distributed parameter systems, Hamiltonian systems, Dirac structures, boundary control.

\section{Mathematics Subject Classification: 70H05, 35B37, 58C20, 93C20}

To be presented at the IFAC Workshop on Lagrangian and Hamiltonian Methods for Nonlinear Control, 16-18 March 2000, Princeton, NJ, USA.

\section{INTRODUCTION}

The Hamiltonian formulation of classes of distributed parameter systems has been a challenging and fruitful area of research for quite some time. A nice introduction, especially with respect to systems stemming from fluid dynamics, can be found in Chapter 7 of the book by Olver (Olver, 1993), where also a historical account is provided. The identification of the underlying Hamiltonian structure of sets of p.d.e.'s has been very instrumental in proving all sorts of results on integrability, the existence of soliton solutions, stability, reduction, etc., and in unifying existing results, see e.g. (Holm et al., 1985).
Recently, there has been also a surge of interest in the control of nonlinear distributed parameter systems, motivated by various applications. At the same time, for finite-dimensional nonlinear systems a satisfactory theory has been developed concerning the generalized Hamiltonian modelling of physical systems with external (input and output) variables. This has led to the notions of portcontrolled Hamiltonian (PCH) systems, and portcontrolled Hamiltonian systems with dissipation (PCHD systems) (van der Schaft and Maschke, 1995), (Dalsmo and van der Schaft, 1999), (Ortega et al., 1999b), (van der Schaft, 2000). This theory is aimed at applications in the consistent modelling and simulation of complex interconnected physical systems, and in the design and con- 
trol of such systems, exploiting the Hamiltonian and passivity structure in a crucial way (van der Schaft, 2000), (Ortega et al., 1999a), (Ortega et al., 1999b).

In the present paper we start to expand the research program on finite-dimensional $\mathrm{PCH}$ and PCHD systems to the distributed parameter (or, infinite-dimensional) case. The first idea for doing so is to try to extend the theory as for instance exposed in (Olver, 1993) to distributed parameter systems with external variables (inputs and outputs). However, a fundamental difficulty which arises is the treatment of boundary conditions. Indeed, from a control and interconnection point of view it is quite essential to describe a distributed parameter system with varying boundary conditions inducing energy exchange through the boundary, since in many applications the interaction with the environment (e.g. actuation or measurement) will actually take place through the boundary of the system. Obvious examples are the telegraphers equations (describing the dynamics of a transmission line), where the boundary of the system is described by the voltages and currents at both ends of the transmission line, or the vibrating string (or, more generally, a flexible beam), where it is natural to consider the forces and velocities at one or both ends of the string as the external variables of the system. On the other hand, the treatment of infinite-dimensional Hamiltonian systems in the literature (see again (Olver, 1993)) seems mostly focussed on systems with infinite spatial domain, where the variables go to zero for the spatial variables tending to infinity, or on systems with boundary conditions such that the energy exchange through the boundary is zero. Furthermore, it is not obvious how to incorporate non-zero energy flow through the boundary in the existing framework. The problem is already illustrated by the Hamiltonian formulation of e.g. the Korteweg-de Vries equation. Here for zero boundary conditions a Poisson bracket can be formulated with the use of the differential operator $\frac{d}{d x}$, since by integration by parts this operator is obviously skew-symmetric. However, for boundary conditions corresponding to nonzero energy flow the differential operator is not skew-symmetric anymore (since the remainders are not zero when integrating by parts). Also the interesting paper (Lewis et al., 1986) does not really solve the problem, since this latter paper is concerned with the modification of the Poisson bracket in case of a free boundary.

In the present paper we propose a framework to overcome this fundamental problem, by defining a Dirac structure on certain spaces of differential forms on the spatial domain and its boundary. This construction of the Dirac structure is based on the use of Stokes' theorem. Then we employ the definition of a port-controlled Hamiltonian system with respect to a Dirac structure, as already given in previous papers (see e.g. (van der Schaft and Maschke, 1995)) for the finite-dimensional case, to describe implicit PCH systems, in order to formalize distributed parameter systems with boundary external variables as infinite-dimensional $\mathrm{PCH}$ systems. This framework is then applied to the port-controlled Hamiltonian formulation of the telegrapher's equations, the vibrating string, and Maxwell's equations on a bounded domain. Due to space limitations the port-controlled Hamiltonian formulation of systems arising in fluid dynamics (e.g. Euler's equations) or in elastodynamics (e.g. flexible beams or plates) will be deferred to a future paper.

\section{DIRAC STRUCTURES AND FINITE-DIMENSIONAL PCHD SYSTEMS}

From a network modeling perspective (Paynter, 1961), (Breedveld, 1984), (Maschke et al., 1992), a lumped parameter physical system is naturally described by a set of (possibly multidimensional) energy-storing elements, a set of energy-dissipating or resistive elements, and a set of ports (by which interaction with the environment can take place), interconnected to each other by a power-conserving interconnection, as depicted in Figure 1.

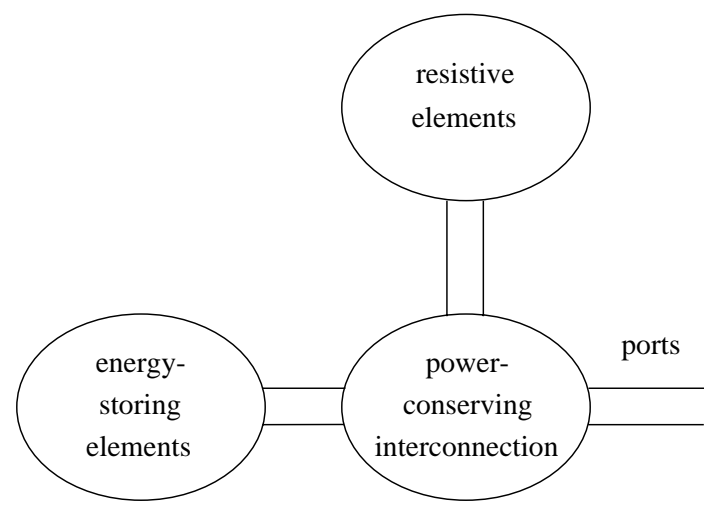

Fig. 1. Implicit port-controlled Hamiltonian system with dissipation

The power-conserving interconnection includes interconnection constraints like Kirchhoff's laws and Newton's third law, as well as power conserving elements like (in the electrical domain) transformers, gyrators, and (in the mechanical domain) transformers, kinematic pairs and kinematic constraints. To every two-fold line (also called power bond) there are associated two kind of variables, usually called flows and efforts, whose product is power. In the mechanical domain the flows and efforts are generalized velocities and forces, while in the electrical domain they are currents 
and voltages. The power-conserving interconnection relates the flows and efforts corresponding to the energy-storing elements, the resistive elements and the ports to each other in such a way that the total incoming power into the interconnection structure is always zero.

Associated with the energy-storing elements are in the lumped parameter case energy-variables $x_{1}, \cdots, x_{n}$, being local coordinates for some $n$ dimensional state space manifold $\mathcal{X}$, and a total energy $H: \mathcal{X} \rightarrow \mathbb{R}$.

The power-conserving interconnection is formalized by a Dirac structure, as was introduced in (Courant, 1990), (Dorfman, 1993). For our purposes in this paper we only need constant Dirac structures on vector spaces. Thus, let $\mathcal{V}$ be (finite or infinite) dimensional linear space, and denote its dual (the space of linear functions on $\mathcal{V}$ ) by $\mathcal{V}^{*}$. The product space $\mathcal{V} \times \mathcal{V}^{*}$ is considered to be the space of flow and effort variables, with power defined by

$$
P=\left\langle v^{*} \mid v\right\rangle, \quad\left(v, v^{*}\right) \in \mathcal{V} \times \mathcal{V}^{*},
$$

where $\left\langle v^{*} \mid v\right\rangle$ denotes the duality product, that is, the linear function $v^{*} \in \mathcal{V}^{*}$ acting on $v \in \mathcal{V}$. Often we call $\mathcal{V}$ the space of flows $f$, and $\mathcal{V}^{*}$ the space of efforts $e$, with the power of an element $(f, e) \in \mathcal{V} \times \mathcal{V}^{*}$ denoted as $\langle e \mid f\rangle$.

Example 1. Let $\mathcal{V}$ be the space of generalized velocities, and $\mathcal{V}^{*}$ be the space of generalized forces, then $\langle e \mid f\rangle$ is mechanical power. Similarly, let $\mathcal{V}$ be the space of currents, and $\mathcal{V}^{*}$ be the space of voltages, then $\langle e \mid f\rangle$ is electrical power.

There exists on $\mathcal{V} \times \mathcal{V}^{*}$ a canonically defined symmetric bilinear form

$$
\left\langle\left\langle\left(f_{1}, e_{1}\right),\left(f_{2}, e_{2}\right)\right\rangle\right\rangle:=\left\langle e_{1} \mid f_{2}\right\rangle+\left\langle e_{2} \mid f_{1}\right\rangle
$$

for $f_{i} \in \mathcal{V}, e_{i} \in \mathcal{V}^{*}, i=1,2$. Now consider a linear subspace

$$
S \subset \mathcal{V} \times \mathcal{V}^{*}
$$

and its orthogonal complement with respect to the bilinear form $\langle\langle\rangle$,$\rangle on \mathcal{V} \times \mathcal{V}^{*}$, denoted as

$$
S^{\perp} \subset \mathcal{V} \times \mathcal{V}^{*}
$$

Definition 1. (Courant, 1990), (Dorfman, 1993), (Dalsmo and van der Schaft, 1999). A constant Dirac structure on $\mathcal{V}$ is a linear subspace $\mathcal{D} \subset \mathcal{V} \times$ $\mathcal{V}^{*}$ such that

$$
\mathcal{D}=\mathcal{D}^{\perp}
$$

Remark 1. In many cases the power-conserving interconnection is actually modulated by the energy variables, in which case the above definition of a constant Dirac structure on a vector space has to be generalized to a general Dirac structure on a manifold, see (Courant, 1990), (Dorfman, 1993).
As an immediate consequence of the definition of a Dirac structure, let $(f, e) \in \mathcal{D}=\mathcal{D}^{\perp}$. Then by (2)

$$
0=\langle\langle(f, e),(f, e)\rangle\rangle=2\langle e \mid f\rangle .
$$

Thus for all $(f, e) \in \mathcal{D}$ we obtain

$$
\langle e \mid f\rangle=0 .
$$

Hence a Dirac structure $\mathcal{D}$ on $\mathcal{V}$ defines a powerconserving relation between the power variables $(f, e) \in \mathcal{V} \times \mathcal{V}^{*}$.

Remark 2. Furthermore, it immediately follows that the dimension of any Dirac structure $\mathcal{D}$ on a finite-dimensional linear space $\mathcal{V}$ is equal to $\operatorname{dim} \mathcal{V}$. This is intimately related to the usually expressed statement that a physical interconnection can not determine at the same time both the flow and effort (e.g. current and voltage, or velocity and force).

It is well-known that a Dirac structure is a generalization both of a symplectic structure and a Poisson structure(Courant, 1990), (Dorfman, 1993), which are the usual geometric building blocks in the definition of a Hamiltonian system. In our previous papers we used Dirac structures in order to also formulate implicit systems as Hamiltonian systems (van der Schaft and Maschke, 1995), (Maschke and van der Schaft, 1996), (Maschke and van der Schaft, 1997), (Maschke and van der Schaft, 1998). Implicit system descriptions naturally arise from network modelling, and the corresponding Dirac structure captures the power conserving interconnection structure of the network. A strongly related argument in favor of the systematic use of Dirac structures in the description of Hamiltonian systems is the fact that the power-conserving interconnection of Dirac structures again defines a Dirac structure (van der Schaft, 1999). In the next section we show how the notion of a Dirac structure (on infinite-dimensional spaces) is also instrumental in the definition of standard distributed parameter systems, which are only implicit in the way the boundary conditions enter the system description.

In order to facilitate the understanding of the definition of Hamiltonian dynamics of distributed parameter systems in the next section we briefly recall the definition of a finite-dimensional implicit PCHD system (van der Schaft and Maschke, 1995), (Maschke and van der Schaft, 1996), (Maschke and van der Schaft, 1997), (Maschke and van der Schaft, 1998), (van der Schaft, 2000). We consider a constant Dirac structure $\mathcal{D}$ on the finite-dimensional linear space $\mathcal{V}:=\mathcal{F}_{S} \times \mathcal{F}_{R} \times \mathcal{F}_{P}$, with $\mathcal{F}_{S}$ denoting the space of flows $f_{S}$ connected to the energy-storing elements, $\mathcal{F}_{R}$ denoting the space of flows $f_{R}$ connected to the dissipative 
(resistive) elements, and $\mathcal{F}_{P}$ the space of external flows $f_{P}$ which can be connected to the environment. Dually, we write $\mathcal{V}^{*}=\mathcal{F}_{S}^{*} \times \mathcal{F}_{R}^{*} \times \mathcal{F}_{P}^{*}$, with $e_{S} \in \mathcal{F}_{S}^{*}$ the efforts connected to the energystoring elements, $e_{R} \in \mathcal{F}_{R}^{*}$ the efforts connected to the resistive elements, and $e_{P} \in \mathcal{F}_{P}^{*}$ the efforts to be connected to the environment of the system. The flow variables of the energy-storing elements are given as $\dot{x}(t)=\frac{d x}{d t}(t), t \in \mathbb{R}$, and the effort variables of the energy-storing elements as $\frac{\partial H}{\partial x}(x(t))$ (implying that $<\frac{\partial H}{\partial x}(x(t)) \mid \dot{x}(t)>=$ $\frac{d H}{d t}(x(t))$ is the increase in energy). In order to have a consistent sign convention for energy flow we put

$$
\begin{aligned}
& f_{S}=-\dot{x} \\
& e_{S}=\frac{\partial H}{\partial x}(x)
\end{aligned}
$$

Then the equations for the implicit PCHD system take the form

$$
\left(-\dot{x}(t), f_{R}(t), f_{P}(t), \frac{\partial H}{\partial x}(x(t)), e_{R}(t), e_{P}(t)\right) \in \mathcal{D},
$$

where the flow and effort variables connected to the resistive elements are related by certain equations

$$
f_{R}=-R\left(e_{R}\right),
$$

where $e_{R}^{T} R\left(e_{R}\right) \geq 0$.

\section{DIRAC STRUCTURE ASSOCIATED WITH THE EXTERIOR DERIVATIVE}

In this section we provide the basic framework for describing distributed parameter systems as portcontrolled Hamiltonian systems. Basic ingredients are the identification of a suitable space of energy variables, closely connected to the geometry of the spatial variables of the distributed parameter system, and the definition of a suitable Dirac structure on the space of energy variables.

In order to define the space of energy variables we first need some preliminaries. Let $N$ be an $n$-dimensional manifold with boundary $\partial N$ (of dimension $n-1)$, representing the space of spatial variables. We denote by $\Omega^{k}(N), k=0,1, \ldots, n$, the space of $k$-forms on $N$, and by $\Omega^{k}(\partial N), k=$ $0,1, \ldots, n-1$, the space of $k$-forms on $\partial N$. (The space $\Omega^{0}(N)$, respectively $\Omega^{0}(\partial N)$, is the space of smooth functions on $N$, respectively $\partial N$.) We note that $\Omega^{k}(N)$ and $\Omega^{k}(\partial N)$ ) are (infinitedimensional) linear spaces with respect to addition and multiplication by elements in $\mathbb{R}$. The dual linear space $\left(\Omega^{k}(N)\right)^{*}$ can be naturally identified with $\Omega^{n-k}(N)$, and similarly the dual space $\left(\Omega^{k}(\partial N)\right)^{*}$ with $\Omega^{n-1-k}(\partial N)$, as stated in the following proposition.
Proposition 1. $\left(\Omega^{k}(N)\right)^{*}$ can be identified with $\Omega^{n-k}(N)$, replacing the duality product between $\Omega^{k}(N)$ and $\left(\Omega^{k}(N)\right)^{*}$ by

$$
\langle\beta \mid \alpha\rangle:=\int_{N} \alpha \wedge \beta, \quad \alpha \in \Omega^{k}(N), \beta \in \Omega^{n-k}(N)
$$

This seems to be a basic fact in the theory of differential forms, although we could not find a reference to this result in the literature. A possible proof for Proposition 1 runs as follows. Equip $N$ with a Riemannian metric (this is always possible), and denote by $\star$ the Hodge star operator. It is well-known (see e.g. Definition 2.7.13 in (Abraham and Marsden, 1978)) that

$$
\left(\alpha_{1}, \alpha_{2}\right):=\int_{N} \alpha_{1} \wedge \star \alpha_{2}, \quad \alpha_{1}, \alpha_{2} \in \Omega^{k}(N),
$$

defines an inner product on $\Omega^{k}(N)$. Then by the Riesz representation theorem there exists for any $\alpha^{*} \in\left(\Omega^{k}(N)\right)^{*}$ a $\gamma \in \Omega^{k}(N)$ such that

$$
\left\langle\alpha^{*} \mid \alpha\right\rangle=(\alpha, \gamma), \quad \alpha \in \Omega^{k}(N)
$$

Denoting $\star \gamma \in \Omega^{n-k}(N)$ by $\beta$ the claimed result follows.

Consider now as space of energy variables the linear space $\mathcal{V}$ defined as follows:

$$
\mathcal{V}:=\Omega^{p}(N) \times \Omega^{q}(N) \times \Omega^{n-q}(\partial N)
$$

for $p$ and $q$ positive integers satisfying

$$
p+q=n+1 \text {. }
$$

By linearity $\mathcal{V}$ is also the space of flows (the rate energy variables). The space $\mathcal{V}$ will be the carrier space for the constant Dirac structure representing the interconnection structure of distributed parameter systems.

Its dual space $\mathcal{V}^{*}$ can be identified as in Proposition 1 with the linear space

$$
\mathcal{V}^{*} \simeq \Omega^{n-p}(N) \times \Omega^{n-q}(N) \times \Omega^{n-p}(\partial N)
$$

(note that $(n-1)-(n-q)=n-p)$. The dual space $\mathcal{V}^{*}$ will represent the space of efforts, or co-energy variables, of the system.

In the present paper we treat for simplicity only the symmetric case $p=q=k$, since this will be sufficient for all the examples treated in Section 4. However, for the treatment of examples from fluid dynamics and elastodynamics it will be necessary to consider the general case, as will be done in a future paper.

Note that in the symmetric case $2 k=n+1$, whence it follows that $n$ is necessarily odd. In fact, the two cases of primary interest for us will be $n=3, k=2$, and $n=1, k=1$. 
Using the identification of Proposition 1 the bilinear form $\langle\langle\cdot, \cdot\rangle\rangle$ on $\mathcal{V} \times \mathcal{V}^{*}$ takes the form

$$
\begin{aligned}
& \left\langle\left\langle\left(f_{E}^{1}, f_{M}^{1}, f_{b}^{1}, e_{E}^{1}, e_{M}^{1}, e_{b}^{1}\right),\left(f_{E}^{2}, f_{M}^{2}, f_{b}^{2}, e_{E}^{2}, e_{M}^{2}, e_{b}^{2}\right)\right\rangle\right\rangle \\
& :=\int_{N}\left(f_{E}^{1} \wedge e_{E}^{2}+f_{M}^{1} \wedge e_{M}^{2}+f_{E}^{2} \wedge e_{E}^{1}+f_{M}^{2} \wedge e_{M}^{1}\right) \\
& +\int_{\partial N}\left(f_{b}^{1} \wedge e_{b}^{2}+f_{b}^{2} \wedge e_{b}^{1}\right) \quad(\in \mathbb{R})
\end{aligned}
$$

with for $i=1,2$

$$
\begin{aligned}
\left(f_{E}^{i}, f_{M}^{i}\right) & \in \Omega^{k}(N) \times \Omega^{k}(N) \\
f_{b}^{i} & \in \Omega^{n-k}(\partial N) \\
\left(e_{E}^{i}, e_{M}^{i}\right) & \in \Omega^{n-k}(N) \times \Omega^{n-k}(N) \\
& \simeq\left(\Omega^{k}(N)\right)^{*} \times\left(\Omega^{k}(N)\right)^{*} \\
e_{b}^{i} & \in \Omega^{n-2-k}(\partial N) \simeq\left(\Omega^{k-1}(\partial N)\right)^{*}
\end{aligned}
$$

The subscripts "E" and "M" here stand for "electric" and "magnetic", corresponding to the two different energy domains in the examples of Maxwell's equations and the telegrapher's equations. However, more generally they will be used as mnemonic notations to denote any two interacting pair of energy domains (as in the vibrating string example, where the energy domains are kinetic and potential energy). Of course, the subscript "b" stands for "boundary".

On $\mathcal{V}$ we now define the following (constant) Dirac structure:

Theorem 2. Define the following linear subspace of $\mathcal{V} \times \mathcal{V}^{*}$

$$
\begin{aligned}
\mathcal{D}= & \left\{\left(f_{E}, f_{M}, f_{b}, e_{E}, e_{M}, e_{b}\right) \in \mathcal{V} \times \mathcal{V}^{*} \mid\right. \\
& {\left[\begin{array}{c}
f_{E} \\
f_{M}
\end{array}\right]=\left[\begin{array}{cc}
0 & (-1)^{n-k} d \\
d & 0
\end{array}\right]\left[\begin{array}{c}
e_{E} \\
e_{M}
\end{array}\right], } \\
& {\left.\left[\begin{array}{l}
f_{b} \\
e_{b}
\end{array}\right]=\left[\begin{array}{cc}
0 & (-1)^{k} \\
1 & 0
\end{array}\right]\left[\begin{array}{c}
\left.e_{E}\right|_{\partial N} \\
\left.e_{M}\right|_{\partial N}
\end{array}\right]\right\} }
\end{aligned}
$$

with $d: \Omega^{p}(N) \rightarrow \Omega^{p+1}(N)$ the usual exterior derivative. Then $\mathcal{D} \subset \mathcal{V} \times \mathcal{V}^{*}$ is a constant Dirac structure.

Proof. (i) $\mathcal{D} \subset \mathcal{D}^{\perp}$ : Let $\left(f_{E}^{1}, f_{M}^{1}, f_{b}^{1}, e_{E}^{1}, e_{M}^{1}, e_{b}^{1}\right) \in$ $\mathcal{D}$ and consider any $\left(f_{E}^{2}, f_{M}^{2}, f_{b}^{2}, e_{E}^{2}, e_{M}^{2}, e_{b}^{2}\right) \in \mathcal{D}$. By substitution of (19) into (17) the right-hand side of (17) becomes

$$
\begin{aligned}
\int_{N}[ & (-1)^{n-k} d e_{M}^{1} \wedge e_{E}^{2}+d e_{E}^{1} \wedge e_{M}^{2} \\
& \left.+(-1)^{n-k} d e_{M}^{2} \wedge e_{E}^{1}+d e_{E}^{2} \wedge e_{M}^{1}\right] \\
+ & \int_{\partial N}(-1)^{k}\left[\left.\left.e_{M}^{1}\right|_{\partial N} \wedge e_{E}^{2}\right|_{\partial N}+\left.\left.e_{M}^{2}\right|_{\partial N} \wedge e_{E}^{1}\right|_{\partial N}\right]
\end{aligned}
$$

From the properties of the exterior derivative it follows that

$$
\begin{aligned}
& d\left(e_{E}^{1} \wedge e_{M}^{2}\right)=d e_{E}^{1} \wedge e_{M}^{2}+(-1)^{n-k} e_{E}^{1} \wedge d e_{M}^{2} \\
& d\left(e_{E}^{2} \wedge e_{M}^{1}\right)=d e_{E}^{2} \wedge e_{M}^{1}+(-1)^{n-k} e_{E}^{2} \wedge d e_{M}^{1}
\end{aligned}
$$

Note that

$$
\begin{aligned}
(-1)^{n-k} e_{E}^{1} & \wedge d e_{M}^{2} \\
& =(-1)^{n-k}(-1)^{(n-k)(n-k+1)} d e_{M}^{2} \wedge e_{E}^{1} \\
& =(-1)^{(n-k)(n-k+2)} d e_{M}^{2} \wedge e_{E}^{1} \\
& =(-1)^{n-k} d e_{M}^{2} \wedge e_{E}^{1}
\end{aligned}
$$

since $(n-k)(n-k+2)$ is odd (respectively even) iff $n-k$ is odd (respectively even). Similarly,

$$
\begin{gathered}
(-1)^{n-k} e_{E}^{2} \wedge d e_{M}^{1}=(-1)^{n-k} d e_{M}^{1} \wedge e_{E}^{2} \\
d\left(e_{E}^{1} \wedge e_{M}^{2}\right)=(-1)^{n-k} d\left(e_{M}^{2} \wedge e_{E}^{1}\right) \\
d\left(e_{E}^{2} \wedge e_{M}^{1}\right)=(-1)^{n-k} d\left(e_{M}^{1} \wedge e_{E}^{1}\right)
\end{gathered}
$$

Substitution of (3),(22) into (21) yields

$$
\begin{aligned}
& (-1)^{n-k} d\left(e_{M}^{2} \wedge e_{E}^{1}\right)= \\
& d e_{E}^{1} \wedge e_{M}^{2}+(-1)^{n-k} d e_{M}^{2} \wedge e_{E}^{1} \\
& (-1)^{n-k} d\left(e_{M}^{1} \wedge e_{E}^{2}\right)= \\
& d e_{E}^{2} \wedge e_{M}^{1}+(-1)^{n-k} d e_{M}^{1} \wedge e_{E}^{2}
\end{aligned}
$$

Finally, by Stokes' theorem

$$
\begin{aligned}
& \int_{N} d\left(e_{M}^{2} \wedge e_{E}^{1}\right)=\left.\left.\int_{\partial N} e_{M}^{2}\right|_{\partial N} \wedge e_{E}^{1}\right|_{\partial N} \\
& \int_{N} d\left(e_{M}^{1} \wedge e_{E}^{2}\right)=\left.\left.\int_{\partial N} e_{M}^{1}\right|_{\partial N} \wedge e_{E}^{2}\right|_{\partial N}
\end{aligned}
$$

Substitution of $(24),(25)$ into (20) yields

$$
\begin{aligned}
& \int_{\partial N}\left[(-1)^{n-k}+(-1)^{k}\right] \\
& \left(\left.\left.e_{M}^{1}\right|_{\partial N} \wedge e_{E}^{2}\right|_{\partial N}+\left.\left.e_{M}^{2}\right|_{\partial N} \wedge e_{E}^{1}\right|_{\partial N}\right.
\end{aligned}
$$

which is zero since for $n$ odd, $(-1)^{n-k}=-(-1)^{k}$. Hence $\left(f_{E}^{1}, f_{M}^{1}, f_{b}^{1}, e_{E}^{1}, e_{M}^{1}, e_{b}^{1}\right) \in \mathcal{D}^{\perp}$.

(ii) $\mathcal{D}^{\perp} \subset \mathcal{D}$ : Let $\left(f_{E}^{1}, f_{M}^{1}, f_{b}^{1}, e_{E}^{1}, e_{M}^{1}, e_{b}^{1}\right) \in \mathcal{D}^{\perp}$, implying that for all $\left(f_{E}^{2}, f_{M}^{2}, f_{b}^{2}, e_{E}^{2}, e_{M}^{2}, e_{b}^{2}\right) \in \mathcal{D}$, (17) equals zero, and hence, by (19) for all

$$
\left((-1)^{n-k} d e_{M}^{2}, d e_{E}^{2},\left.(-1)^{k} e_{M}^{2}\right|_{\partial N}, e_{E}^{2}, e_{M}^{2},\left.e_{E}^{2}\right|_{\partial N}\right)
$$

Therefore for all $e_{E}^{2}, e_{M}^{2}$ :

$$
\begin{aligned}
& \int_{N}\left(f_{E}^{1} \wedge e_{E}^{2}+f_{M}^{1} \wedge e_{M}^{2}+\right. \\
& \left.(-1)^{n-k} d e_{M}^{2} \wedge e_{E}^{1}+d e_{E}^{2} \wedge e_{M}^{1}\right)+ \\
& (-1)^{k} \int_{\partial N}\left(\left.f_{b}^{1} \wedge e_{E}^{2}\right|_{\partial N}+\left.e_{M}^{2}\right|_{\partial N} \wedge e_{b}^{1}\right)=0
\end{aligned}
$$

Now, first consider $(n-k)$-forms $e_{E}^{1}, e_{M}^{2}$ with $\left.e_{E}^{2}\right|_{\partial N}=\left.e_{M}^{2}\right|_{\partial N}=0$. Then the second term of (28) automatically vanishes. Substitution of (24) 
into the first term of (28) now yields by Stokes' theorem (since $\left.\left.e_{E}^{2}\right|_{\partial N}=\left.e_{M}^{2}\right|_{\partial N}=0\right)$

$$
\begin{aligned}
& \int_{N}\left(f_{E}^{1} \wedge e_{E}^{2}+f_{M}^{1} \wedge e_{M}^{2}-d e_{E}^{1} \wedge e_{M}^{2}-\right. \\
&\left.(-1)^{n-k} d e_{M}^{1} \wedge e_{E}^{2}\right)
\end{aligned}
$$

for all $e_{E}^{2}, e_{M}^{2}$ with $\left.e_{E}^{2}\right|_{\partial N}=\left.e_{M}^{2}\right|_{\partial N}=0$. Hence

$$
\begin{aligned}
& \int_{N}\left(f_{E}^{1}-(-1)^{n-k} d e_{M}^{1}\right) \wedge e_{E}^{2}=0 \\
& \int_{N}\left(f_{M}^{1}-d e_{E}^{1}\right) \wedge e_{M}^{2}=0
\end{aligned}
$$

for all $e_{E}^{2}, e_{M}^{2}$ with $\left.e_{E}^{2}\right|_{\partial N}=\left.e_{M}^{2}\right|_{\partial N}=0$. This implies

$$
f_{E}^{1}=(-1)^{n-k} d e_{M}^{1}, \quad f_{M}^{1}=d e_{E}^{1}
$$

Substitution of (31) into (28) then yields, after application of $(24),(25)$,

$$
\begin{aligned}
& (-1)^{n-k} \int_{\partial N}\left(\left.\left.e_{M}^{1}\right|_{\partial N} \wedge e_{E}^{2}\right|_{\partial N}+\left.\left.e_{M}^{2}\right|_{\partial N} \wedge e_{E}^{1}\right|_{\partial N}\right) \\
& +\int_{N}\left(\left.f_{b}^{1} \wedge e_{E}^{2}\right|_{\partial N}+\left.(-1)^{k} e_{M}^{2}\right|_{\partial N} \wedge e_{b}^{1}\right)=0
\end{aligned}
$$

for all $\left.e_{E}^{2}\right|_{\partial N},\left.e_{M}^{2}\right|_{\partial N}$, showing that

$$
\begin{array}{r}
f_{b}^{1}=-\left.(-1)^{n-k} e_{M}^{1}\right|_{\partial N} \\
(-1)^{k} e_{b}^{1}=-\left.(-1)^{n-k} e_{E}^{1}\right|_{\partial N}
\end{array}
$$

or equivalently (since $n$ is odd)

$$
f_{b}^{1}=\left.(-1)^{k} e_{M}^{1}\right|_{\partial N}, \quad e_{b}^{1}=\left.e_{E}^{1}\right|_{\partial N}
$$

Thus, (31) together with (34) yields $\left(f_{E}^{1}, f_{M}^{1}, f_{b}^{1}\right.$, $\left.e_{E}^{1}, e_{M}^{1}, e_{b}^{1}\right) \in \mathcal{D}$, showing that $\mathcal{D}^{\perp} \subset \mathcal{D}$.

Remark 3. Note that for the two cases of primary interest $(n=3, k=2$, and $n=1, k=1)$ the Dirac structure $\mathcal{D}$ amounts for $n=3, k=2$ to

$$
\begin{gathered}
{\left[\begin{array}{c}
f_{E} \\
f_{M}
\end{array}\right]=\left[\begin{array}{cc}
0 & -d \\
d & 0
\end{array}\right]\left[\begin{array}{c}
e_{E} \\
e_{M}
\end{array}\right],} \\
{\left[\begin{array}{c}
f_{b} \\
e_{b}
\end{array}\right]=\left[\begin{array}{ll}
0 & 1 \\
1 & 0
\end{array}\right]\left[\begin{array}{c}
\left.e_{E}\right|_{\partial N} \\
\left.e_{M}\right|_{\partial N}
\end{array}\right]}
\end{gathered}
$$

and for $n=1, k=1$ to:

$$
\begin{gathered}
{\left[\begin{array}{l}
f_{E} \\
f_{M}
\end{array}\right]=\left[\begin{array}{ll}
0 & d \\
d & 0
\end{array}\right]\left[\begin{array}{c}
e_{E} \\
e_{M}
\end{array}\right],} \\
{\left[\begin{array}{c}
f_{b} \\
e_{b}
\end{array}\right]=\left[\begin{array}{cc}
0 & -1 \\
1 & 0
\end{array}\right]\left[\begin{array}{l}
\left.e_{E}\right|_{\partial N} \\
\left.e_{M}\right|_{\partial N}
\end{array}\right]}
\end{gathered}
$$

Remark 4. The vanishing of the bilinear form (17) restricted to $\mathcal{D}$ should be interpreted as a generalized form of power conservation. Indeed, for

$$
\begin{aligned}
\left(f_{E}^{i}, f_{M}^{i}, f_{b}^{i}, e_{E}^{i}, e_{M}^{i}, e_{b}^{i}\right) & \\
= & \left(f_{E}, f_{M}, f_{b}, e_{E}, e_{M}, e_{b}\right) \in \mathcal{D}
\end{aligned}
$$

$i=1,2$, we obtain

$$
\int_{N}\left(f_{E} \wedge e_{E}+f_{M} \wedge e_{M}\right)+\int_{\partial N}\left(f_{b} \wedge e_{b}\right)=0
$$

The first term in (37) represents incoming power via the energy-storing elements in the domain $N$, while the second term represents the incoming power (originating from the environment) via the boundary $\partial N$.

Remark 5. The compositionality properties of the Dirac structure defined in Theorem 2 follow immediately. Indeed, consider two manifolds with boundary $N_{1}$ and $N_{2}$, such that

$$
\begin{array}{ll}
\partial N_{1}=\Gamma \cup \Gamma_{1}, & \Gamma \cap \Gamma_{1}=\emptyset \\
\partial N_{2}=\Gamma \cup \Gamma_{2}, & \Gamma \cap \Gamma_{2}=\emptyset
\end{array}
$$

(that is, $N_{1}$ and $N_{2}$ have boundary $\Gamma$ in common). Then the Dirac structures $\mathcal{D}_{1}, \mathcal{D}_{2}$ on $N_{1}$, respectively $N_{2}$, compose to the Dirac structure $\mathcal{D}$ on $N=N_{1} \cup N_{2}$ with boundary $\Gamma_{1} \cup \Gamma_{2}$, if we equate $f_{b}^{1}$ on $\Gamma$ with $-f_{b}^{2}$ on $\Gamma$, and $e_{b}^{1}$ on $\Gamma$ with $e_{b}^{2}$ on $\Gamma$. (That is, the power flowing into $N_{1}$ via $\Gamma$ should be equal to the power flowing out of $N_{2}$ via $\Gamma$.)

The definition of a distributed parameter portcontrolled Hamiltonian systems now follows immediately. Consider a Hamiltonian density (energy per volume element)

$$
H: \Omega^{k}(N) \times \Omega^{k}(N) \times N \rightarrow \Omega^{n}(N)
$$

resulting in the total energy

$$
\mathcal{H}:=\int_{N} H \quad \in \mathbb{R}
$$

We throughout assume $H$ to be differentiable, with gradient vector denoted as

$$
\operatorname{grad} H=\left(\delta_{E} H, \delta_{M} H\right) \in\left(\Omega^{k}(N) \times \Omega^{k}(N)\right)^{*}
$$

Using the identification of Proposition 1 we thus obtain the co-energy variables

$$
\begin{aligned}
& \delta_{E} H \in \Omega^{n-k}(N) \\
& \delta_{M} H \in \Omega^{n-k}(N)
\end{aligned}
$$

Now, consider time-functions

$$
\left(\alpha_{E}(t), \alpha_{M}(t)\right) \in \Omega^{k}(N) \times \Omega^{k}(N), \quad t \in \mathbb{R}
$$

and the Hamiltonian density $H\left(\alpha_{E}(t), \alpha_{M}(t)\right), t \in$ $\mathbb{R}$, evaluated along this trajectory. It follows that 


$$
\begin{aligned}
\frac{d H}{d t} & =\langle\operatorname{grad} H|\left(\frac{\partial \alpha_{E}}{\partial t}, \frac{\partial \alpha_{M}}{\partial t}\right\rangle \\
& =\frac{\partial \alpha_{E}}{\partial t} \wedge \delta_{E} H+\frac{\partial \alpha_{M}}{\partial t} \wedge \delta_{M} H
\end{aligned}
$$

and hence for the total energy $\mathcal{H}$,

$$
\frac{d \mathcal{H}}{d t}=\int_{N}\left(\frac{\partial \alpha_{E}}{\partial t} \wedge \delta_{E} H+\frac{\partial \alpha_{M}}{\partial t} \wedge \delta_{M} H\right)
$$

The $k$-forms $\frac{\partial \alpha_{E}}{\partial t}$, $\frac{\partial \alpha_{M}}{\partial t}$ represent the (infinitedimensional) generalized velocities corresponding to the energy storage in $N$. They are connected to the Dirac structure $D$ by setting (analogous to (8))

$$
\begin{aligned}
& f_{E}=-\frac{\partial \alpha_{E}}{\partial t} \\
& f_{M}=-\frac{\partial \alpha_{M}}{\partial t}
\end{aligned}
$$

(The minus sign is included in order to have a consistent energy flow description.) Furthermore, we set (analogously to (8))

$$
\begin{aligned}
& e_{E}=\delta_{E} H \\
& e_{M}=\delta_{M} H
\end{aligned}
$$

Definition 2. The distributed parameter portcontrolled Hamiltonian system with manifold of spatial variables $N$, state space $\Omega^{k}(N) \times \Omega^{k}(N)$, Dirac structure $D$ on $\Omega^{k}(N) \times \Omega^{k}(N) \times \Omega^{k-1}(\partial N)$ given by (19), and Hamiltonian density $H$, is given as

$$
\begin{aligned}
{\left[\begin{array}{r}
-\frac{\partial \alpha_{E}}{\partial t} \\
-\frac{\partial \alpha_{M}}{\partial t}
\end{array}\right] } & =\left[\begin{array}{cc}
0 & (-1)^{n-k} d \\
d & 0
\end{array}\right]\left[\begin{array}{l}
\delta_{E} H \\
\delta_{M} H
\end{array}\right] \\
{\left[\begin{array}{l}
f_{b} \\
e_{b}
\end{array}\right] } & =\left[\begin{array}{cc}
0 & (-1)^{k} \\
1 & 0
\end{array}\right]\left[\begin{array}{l}
\delta_{E} H \\
\delta_{M} H
\end{array}\right]
\end{aligned}
$$

with $f_{b}, e_{b} \in \Omega^{k-1}(\partial N)$ denoting the boundary variables.

Note that (48) defines a (nonlinear) boundary control system in the sense of e.g. (Fattorini, 1968), with inputs, say, $f_{b}$, and outputs $e_{b}$. It immediately follows from (45) and the powerconservation property of the Dirac structure (see Remark 4) that any distributed parameter portcontrolled Hamiltonian system satisfies along its trajectories the energy-balance

$$
\frac{d \mathcal{H}}{d t}=\int_{\partial N} f_{b} \wedge e_{b},
$$

expressing that the increase in internally stored energy in $N$ equals the incoming power via the boundary $\partial N$.

Energy exchange through the boundary is not the only way a distributed parameter system may interact with its environment. An example of a different situation is formed by Maxwell's equations (see Section 4.2), where interaction may also take place via the current density $J$ which directly affects the electric charge distribution in the domain $N$. In order to cope with this other possibility we extend the space $\mathcal{V}$ given by

$$
\Omega^{k}(N) \times \Omega^{k}(N) \times \Omega^{k-1}(\delta N)
$$

to

$$
\Omega^{k}(N) \times \Omega^{k}(N) \times \Omega^{k-1}(\delta N) \times \Omega^{k}(N)
$$

with the last component denoting the external distributed flow $f_{d} \in \Omega^{k}(N)$, with dual variable the distributed effort $e_{d} \in \Omega^{n-k}(N)$. In this case, the first line of the definition of the Dirac structure given in Theorem 2 is extended to

$$
\begin{aligned}
{\left[\begin{array}{c}
f_{E} \\
f_{M}
\end{array}\right] } & =\left[\begin{array}{cc}
0 & (-1)^{n-k} d \\
d & 0
\end{array}\right]\left[\begin{array}{c}
e_{E} \\
e_{M}
\end{array}\right]+G f_{d} \\
e_{d} & =G^{*}\left[\begin{array}{c}
e_{E} \\
e_{M}
\end{array}\right]
\end{aligned}
$$

for some linear map $G: \Omega^{k}(N) \rightarrow \Omega^{k}(N) \times$ $\Omega^{k}(N)$, with dual map $G^{*}: \Omega^{n-k}(N) \times \Omega^{n-k}(N) \rightarrow$ $\Omega^{n-k}(N)$. If the map $G$ is modulated by the state variables $\alpha_{E}, \alpha_{M}$, then the resulting Dirac structure is not constant anymore. The port variables are now $\left(f_{b}, f_{d}, e_{b}, e_{d}\right)$, with $f_{b}, e_{b}$ the boundary port variables, and $f_{d}, e_{d}$ the distributed port variables. Finally, energy dissipation can be incorporated in the framework by terminating some of the ports (boundary or distributed) with a resistive relation as in (10).

\section{ELECTRODYNAMICS AND THE VIBRATING STRING}

\subsection{The telegrapher's equations}

In this example we shall consider the telegrapher's equations, i.e. the transverse electromagnetic wave propagating in the dielectric of an ideal lossless transmission line (Dworsky, 1979). Let us first briefly recall the classical formulation of the telegrapher's equations using real functions. The spatial variable $z$ belongs to some segment, for instance $N=[0, L]$ of the real line $\mathbb{R}$. The energy variables are the charge per unit length $q(z, t)$ and the magnetic flux per unit length $\phi(z, t)$. The lossless transmission line is characterized by the distributed capacitance $C(z)$ and the distributed inductance $L(z)$ which defines the electromagnetic energy density:

$$
E(q, \phi, z)=\frac{1}{2} \frac{q^{2}}{C}+\frac{1}{2} \frac{\phi^{2}}{L}
$$

The coenergy variables are hence the voltage $V(z)$ : 


$$
V(z)=\frac{\partial H}{\partial q}=\frac{q}{C}
$$

and the current $I(z)$ :

$$
I(z)=\frac{\partial H}{\partial \phi}=\frac{\phi}{L}
$$

Then Maxwell's equations in the case of a planar transverse electromagnetic wave become the telegrapher's equations:

$$
\begin{aligned}
& \frac{\partial q}{\partial t}=-\frac{\partial}{\partial z} I(z) \\
& \frac{\partial \phi}{\partial t}=-\frac{\partial}{\partial z} V(z)
\end{aligned}
$$

In the formalism of port controlled Hamiltonian systems with power flow through the boundaries proposed in Section 3, the telegrapher's equations are formulated as follows. The energy variables (electric charge and magnetic flux) are the 1forms:

$$
\begin{aligned}
& \alpha_{E}(t)=q(z, t) d z \in \Omega^{1}([0, L]) \\
& \alpha_{M}(t)=\phi(z, t) d z \in \Omega^{1}([0, L])
\end{aligned}
$$

The co-energy variables (voltage and current) are the following 0 -forms, (functions) $V(z) \in$ $\Omega^{0}([0, L])$ and $I(z) \in \Omega^{0}([0, L])$ which are related to the energy variables using the Hodge star product (associated with the canonical inner product on $\mathbb{R}$ ) and the characteristic capacitance and inductance of the line, as follows:

$$
\begin{aligned}
& V=\frac{1}{C} \star \alpha_{E} \\
& I=\frac{1}{L} \star \alpha_{M}
\end{aligned}
$$

Then the energy density $E$ in (53) becomes the following one-form:

$$
H_{t l}\left(\alpha_{E}, \alpha_{M}\right)=\frac{1}{2}\left(\alpha_{E} \wedge V+\alpha_{M} \wedge I\right)
$$

which by definition of the Hodge star operator * may be expressed as a quadratic form on $\alpha_{E}$ and $\alpha_{M}$. Moreover, by construction, the energy density $H_{t l}$ in (59) satisfies Maxwell's reciprocity conditions. The total electromagnetic energy of the transmission line is then:

$$
\mathcal{H}_{t l}=\int_{N} H_{t l}
$$

Then the telegrapher's equations may be expressed as a distributed port controlled Hamiltonian system according to definition 2 with power flow through the boundary $\delta N=\{0, L\}$ of $N$ and boundary port variables being the current at the terminal points of the line: $f_{b}=-\left.I\right|_{\delta N}$ and the voltage: $e_{b}=\left.V\right|_{\delta N}$. This port controlled Hamiltonian system is defined with respect to the Dirac structure defined in Theorem 2 with $n=k=1$, and the Hamiltonian functional being the total electromagnetic energy defined in (59) and (60).

The power balance (49) becomes:

$$
\frac{d \mathcal{H}_{t l}}{d t}=\int_{\delta N} f_{b} \wedge e_{b}=V(0) I(0)-V(L) I(L)
$$

and says that the time derivative of the total magnetic energy is equal to the electromagnetic power ingoing the line at the point 0 minus the electromagnetic power outgoing the line at $L$.

\subsection{Maxwell's equations}

In this paragraph we shall briefly present the port controlled Hamiltonian description of Maxwell's equations following closely the formulation in terms of differential forms presented in (Ingarden and Jamiolkowski, 1985).

We consider some connected closed domain $N$ with non void interior, of the three-dimensional oriented Euclidean space $E^{3}$ which defines the spatial variable $x \in N$ and consider the electromagnetic field in the medium in $N$.

The energy variables are the electric field induction 2-forms $\alpha_{E}=\mathcal{D} \in \Omega^{2}(N)$ :

$$
\mathcal{D}=\frac{1}{2} D_{i j}(x, t) d x^{i} \wedge d x^{j}
$$

and the magnetic field induction 2-form $\alpha_{M}=$ $\mathcal{B} \in \Omega^{2}(N):$

$$
\mathcal{B}=\frac{1}{2} B_{i j}(x, t) d x^{i} \wedge d x^{j}
$$

Note that using the Hodge star operator the two 2-forms may be transformed to 1-forms usually called electric field induction vector $\star \mathcal{D}=$ $D_{i}(x, t) d x^{i}$ and the magnetic field induction vector $\star \mathcal{B}=B_{i}(x, t) d x^{i}$.

The coenergy variables are the electric field intensity 1-forms $\mathcal{E} \in \Omega^{1}(N)$ :

$$
\mathcal{E}=E_{i}(x, t) d x^{i}
$$

and the magnetic field intensity 1-forms $\mathcal{H} \in$ $\Omega^{1}(N)$ :

$$
\mathcal{H}=H_{i}(x, t) d x^{i}
$$

These two 1-forms are usually called vectors of electric and magnetic field intensity.

The constitutive relations of the medium define the relations between the coenergy and the energy variables:

$$
\begin{aligned}
& \mathcal{E}=\epsilon^{-1} \star \mathcal{D} \\
& \mathcal{H}=\mu^{-1} \star \mathcal{B}
\end{aligned}
$$


where $\epsilon(x)$ denotes the (symmetric positive) electric permittivity tensor and $\mu(x)$ denotes the (symmetric positive) magnetic permittivity tensor.

Hence the electromagnetic energy density 3-form $\mathcal{H}_{e} m \in \Omega^{3}(N)$ becomes:

$$
H_{\text {em }}(\mathcal{D}, \mathcal{B})=\frac{1}{2}(\mathcal{E} \wedge \mathcal{D}+\mathcal{H} \wedge \mathcal{B})
$$

One may note again that, by definition of the Hodge star product, the electromagnetic energy density may be expressed as a quadratic form on $\mathcal{D}$ and $\mathcal{B}$. Moreover, by symmetry of the electric and magnetic permittivity tensors, the energy density $H_{e m}$ in (67) satisfies Maxwell's reciprocity conditions. The total electromagnetic energy in the medium in $N$ is then:

$$
\mathcal{H}_{e m}=\int_{N} H_{e m}
$$

Assuming that there is no current in the medium, Maxwell's equations may then be written as follows:

$$
\begin{aligned}
\frac{\partial}{\partial t} \mathcal{D} & =d \mathcal{H} \\
\frac{\partial}{\partial t} \mathcal{B} & =-d \mathcal{E}
\end{aligned}
$$

Note that the $d$ denotes the exterior derivative and is applied to 1 -forms in $\Omega^{1}(N)$. Using the Hodge star product and the resulting identification of 1 and 2-forms with vectors, the exterior derivative is then simply the curl of a vector.

Using the definition of the energy and coenergy variables as differential forms, Maxwell's equations may hence be expressed as a distributed parameter port controlled Hamiltonian system according to Definition 2, with power flow through the boundary $\delta N$ of $N$ and boundary port variables being the electric field intensity at the boundary: $\delta N f_{b}=\left.\mathcal{E}\right|_{\delta N}$ and the magnetic field intensity at the boundary $\delta N: e_{b}=\left.\mathcal{H}\right|_{\delta N}$. This port controlled Hamiltonian system is defined with respect to the Dirac structure defined in Theorem 2 with $n=3$ and $k=2$, and is generated by the Hamiltonian functional being the total electromagnetic energy defined in (67) and (68).

The power balance (49) becomes:

$$
\frac{d \mathcal{H}_{t l}}{d t}=\int_{\delta N} f_{b} \wedge e_{b}=\int_{\delta N} \mathcal{E} \wedge \mathcal{H}
$$

and says that the time derivative of the total electromagnetic energy in $N$ is equal to the flow of electromagnetic power radiating through the boundary $\delta N$. It may be noted that the power through the boundary is defined by the 2 -form $\mathcal{S} \in \Omega^{2}(N)$, the so-called Poynting vector:

$$
\mathcal{S}=\mathcal{E} \wedge \mathcal{H}
$$

Assuming that there exists a current density in the domain $N$, for instance if the medium is conducting due to Ohm's law or due to the diffusion of the charges or to some temperature gradient, then there exist an energy exchange with some other physical domain than the electromagnetic field, for instance with the thermic domain. This energy exchange does not go through the boundary of the domain $N$ but is distributed in $N$. The current density may be defined as a 2-form $\mathcal{J} \in \Omega^{2}(N)$ which is related to the classically defined current density vector by the Hodge star operator: $\star \mathcal{J}=J_{i}(x, t) d x^{i}$. The dynamics of the electromagnetic field is now described by the following port controlled Hamiltonian system with power exchange through the boundary and in the domain:

$$
\begin{aligned}
{\left[\begin{array}{l}
\frac{\partial \mathcal{D}}{\partial t} \\
\frac{\partial \mathcal{B}}{\partial t}
\end{array}\right] } & =\left[\begin{array}{cc}
0 & d \\
-d & 0
\end{array}\right]\left[\begin{array}{l}
\delta_{\mathcal{D}} H_{e m} \\
\delta_{\mathcal{B}} H_{e m}
\end{array}\right]+\left[\begin{array}{l}
1 \\
0
\end{array}\right] \mathcal{J} \\
{\left[\begin{array}{l}
f_{b} \\
e_{b}
\end{array}\right] } & =\left[\begin{array}{ll}
0 & 1 \\
1 & 0
\end{array}\right]\left[\begin{array}{l}
\left.\delta_{\mathcal{D}} H_{e m}\right|_{\delta N} \\
\left.\delta_{\mathcal{B}} H_{e m}\right|_{\delta N}
\end{array}\right] \\
e_{d} & =\left[\begin{array}{ll}
1 & 0
\end{array}\right]\left[\begin{array}{l}
\delta_{\mathcal{D}} H_{e m} \\
\delta_{\mathcal{B}} H_{e m}
\end{array}\right]=\mathcal{E}
\end{aligned}
$$

One may note that the conjugated port variable to the current density is simply the electric intensity 2-form: $e_{d}=\mathcal{E}$.

The power balance (70) becomes:

$$
\frac{d \mathcal{H}_{t l}}{d t}=\int_{\delta N} \mathcal{E} \wedge \mathcal{H}+\int_{N} \mathcal{E} \wedge \mathcal{J}
$$

and says that the variation of the total electromagnetic energy in $N$ is equal to the flow of electromagnetic power radiating through the boundary $\delta N$ plus the power exchanged in the volume (for instance dissipated by Ohm's law in the case of a conducting medium).

\subsection{Vibrating string}

Consider now an elastic string subject to traction forces at its ends. The spatial variable $z$ belong to some segment, for instance $N=[0, L]$ of the real line $\mathbb{R}$. The dynamics of the string arise from the interaction of the elastic-potential energy and the kinetic energy of the string. Let us denote by $u(z, t)$ the displacement of the string. The elastic potential energy is a function of the strain variable, the 1-form:

$$
\alpha_{E}(t)=\epsilon(z, t) d z \in \Omega^{1}([0, L])
$$

where $\epsilon(z, t)=\frac{\partial}{\partial z} u(z, t)$.

The associated coenergy variable is the stress variable which is the 0 -form (function) $\sigma(z) \in$ $\Omega^{0}([0, L])$, which is related to the strain variable 
$\alpha_{E}$ using the Hodge star operator (associated with the canonical inner product on $\mathbb{R}$ ) and the characteristic elasticity modulus $T$ :

$$
\sigma=T \star \alpha_{E}
$$

The kinetic energy is defined through the energy variable given as the kinetic momentum, which is a 1-form:

$$
\alpha_{M}(t)=p(z, t) d z \in \Omega^{1}([0, L])
$$

with the co-energy variable being the velocity $v(z, t)=\frac{\partial}{\partial t} u(z, t)$ at $z$, interpreted as a 0 -form $v \in \Omega^{0}([0, L])$, related to the kinetic momentum by:

$$
v=\frac{1}{\mu} \star \alpha_{E}
$$

where $\mu$ is the mass density of the string.

Then the total energy density (sum of the elastic potential and kinetic energy density) is then the following one-form:

$$
H_{\text {string }}\left(\alpha_{E}, \alpha_{M}\right)=\frac{1}{2}\left(\alpha_{E} \wedge \sigma+\alpha_{M} \wedge v\right)
$$

which by definition of the Hodge star product may be expressed as a quadratic form on $\alpha_{E}$ and $\alpha_{M}$. The total energy of the string is then:

$$
\mathcal{H}_{\text {string }}=\int_{N} H_{\text {string }}
$$

The elastodynamic equations of the string may be expressed as a distributed port controlled Hamiltonian system according to Definition 2 with power flow through the boundary $\delta N=\{0, L\}$ of $N$ and boundary port variables being the stress at the terminal points of the line: $f_{b}=-\left.\sigma\right|_{\delta N}$ and the velocity: $e_{b}=\left.v\right|_{\delta N}$. This port controlled Hamiltonian system is defined with respect to the Dirac structure defined in Theorem 2 with $n=k=1$ and the Hamiltonian functional being the total energy of the string defined in (78) and (79).

The power balance (49) becomes:

$$
\frac{d \mathcal{H}_{t l}}{d t}=\int_{\delta N} f_{b} \wedge e_{b}=v(0) \sigma(0)-v(L) \sigma(L)
$$

and says that the time derivative of the total magnetic energy is equal to the balance of the mechanical work at the points 0 and $L$.

\section{CONCLUSION}

We have presented a port controlled Hamiltonian formulation of the dynamics of distributed parameter systems which allows to represent the energy flows through the boundary of the domain of the system.

The state space of the distributed port controlled Hamiltonian system is a space of differential forms on the domain of the spatial variables and corresponds to the energy variables of the system. The boundary variables are similarly defined as differential forms on the boundary of the domain. From this geometric definition of the state variables and the boundary variables, we have derived a Dirac structure associated with the exterior derivative and based on Stokes' theorem.

Then the dynamics of the distributed parameter system was formulated as an implicit port controlled Hamiltonian system defined with respect to this Dirac structure. This was illustrated on the examples of the telegrapher's equations, Maxwell's equations and the vibrating string.

In this paper we have restricted the presentation to the symmetric case where the state space consists of the product space of differential forms of the same degree. However the construction may be generalized in a straightforward way to the asymmetric case which arises for instance in the dynamics of compressible fluids, as will be presented in a forthcoming paper. The same examples also necessitate to consider Dirac structures not only derived from the exterior derivative but augmented with a Lie-Poisson bracket. Furthermore, Dirac structures offer an ideal frame to treat geometrically constrained distributed parameter systems as they appear in models of flexible beams (Saintellier, 1993).

Finally, an important goal of the Hamiltonian formulation, apart from the system theoretic, modelling and simulation purposes, is the design of passivity-based and physically interpretable stabilizing control stategies for distributed parameter systems using an extension of control schemes proposed for finite-dimensional systems (Ortega et al., 1999a; Ortega et al., 1999b).

\section{REFERENCES}

Abraham, R. and J.E. Marsden (1978). Foundations of Mechanics. second ed.. Benjamin/Cummings Publishing Company.

Breedveld, P.C. (1984). Physical Systems Theory in Terms of Bond Graphs. PhD thesis. Technische Hogeschool Twente. Enschede, The Netherlands.

Courant, T. (1990). Dirac manifolds. Trans. American Math. Soc. 319, 631-661.

Dalsmo, M. and A.J. van der Schaft (1999). On representations and integrability of mathematical structures in energy-conserving physical systems. SIAM J. Cont. Opt. 37(1), 5491.

Dorfman, I. (1993). Dirac Structures and Integrability of Nonlinear Evolution Equations. Chichester: John Wiley. 
Dworsky, L.N. (1979). Modern Transmission Line Theory and Applications. Wiley.

Fattorini, H.O. (1968). Boundary control systems. SIAM J. Control 6, 349-385.

Holm, D.D., J.E. Marsden, T.E. Ratiu and A. Weinstein (1985). Nonlinear stability of fluid and plasma equilibria. Phys.Rep. 123, 1-116.

Ingarden, R.S. and A. Jamiolkowski (1985). Classical Electrodynamics. PWN-Polish Sc. Publ., Warszawa, Elsevier.

Lewis, D., J.E. Marsden, R. Montgomery and R. Ratiu (1986). The Hamiltonian structure for dynamic free boundary problems. Physica D 18, 391-404.

Maschke, B.M., A.J. van der Schaft and P.C. Breedveld (1992). An intrinsic Hamiltonian formulation of network dynamics: Nonstandard poisson structures and gyrators. Journal of the Franklin Institute 329(5), 923966.

Maschke, B.M. and A.J. van der Schaft (1996). Interconnection of systems: the network paradigm. In: Proc. of the IEEE Int. Conf. on Decision and Control, CDC'96. Kobe, Japan. pp. 207-212.

Maschke, B.M. and A.J. van der Schaft (1997). Interconnected mechanical systems, part II: the dynamics of spatial mechanical networks. In: Modelling and Control of Mechanical Systems (A. Astolfi, D.J.N Limebeer, C. Melchiorri, A. Tornambè and R.B. Vinter, Eds.). Imperial College Press. pp. 17-30.

Maschke, B.M. and A.J. van der Schaft (1998). Note on the dynamics of LC circuits with elements in excess. Memorandum 1426. University of Twente. Faculty of Applied Mathematics.

Olver, P.J. (1993). Applications of Lie Groups to Differential Equations. second ed.. SpringerVerlag.

Ortega, R., A.J. van der Schaft and B.M Maschke (1999a). Stabilization of port controlled Hamiltonian systems. In: Stability and Stabilization of Nonlinear Systems (D.Aeyels, F.Lamnabhi-Lagarrigue and A.J. van der Schaft, Eds.). Vol. 246 of LNCIS. Springer. pp. 239-260.

Ortega, R., A.J. van der Schaft, B.M. Maschke and G. Escobar (1999b). Stabilization of portcontrolled Hamiltonian systems: Passivation and energy-balancing. Submitted to Automatica. University of Twente.

Paynter, H. M. (1961). Analysis and Design of Engineering Systems. M.I.T. Press. Cambridge, Massachusetts.

Saintellier, F. (1993). Modelling and Simulation of Robots with Flexible Links using Bond Graphs for their Control (in french). Master's thesis. Conservatoire National des Arts et Métiers. Paris, France. van der Schaft, A.J. (1999). Interconnection and Geometry. In: From Intelligent Control to Behavioral Systems (J.W. Polderman and H.L. Trentelman, Eds.). Groningen. pp. 203-218.

van der Schaft, A.J. (2000). L $L_{2}$-Gain and Passivity Techniques in Nonlinear Control. Communications and Control Engineering. 2nd revised edition, Springer-Verlag.

van der Schaft, A.J. and B.M Maschke (1995). The Hamiltonian formulation of energy conserving physical systems with external ports. Archiv für Elektronik und Übertragungstechnik 49, 362-371. 